\title{
Vascular-Streak Dieback: A New Encounter Disease of Cacao in Papua New Guinea and Southeast Asia Caused by the Obligate Basidiomycete Oncobasidium theobromae
}

\author{
David Guest and Philip Keane
}

First author: The University of Sydney, NSW 2006, Australia; and second author: La Trobe University, Victoria 3086, Australia.

\begin{abstract}
Guest, D., and Keane, P. 2007. Vascular-streak dieback: A new encounter disease of cacao in Papua New Guinea and Southeast Asia caused by the obligate basidiomycete Oncobasidium theobromae. Phytopathology 97: 1654-1657.

The basidiomycete Oncobasidium theobromae was identified as the cause of a devastating disease of cacao named vascular-streak dieback (VSD) in Papua New Guinea in the 1960s. VSD now causes losses among cacao seedlings and kills branches in mature cacao trees throughout Southeast Asia and parts of Melanesia. The characteristic symptoms include a green-spotted chlorosis and fall of leaves beginning on the second or third flush behind the stem apex, raised lenticels, and darkening of vascular traces at the leaf scars and infected xylem. Eventually complete

defoliation occurs and, if the fungus spreads to the trunk, the tree will die. $O$. theobromae is a highly specialized, near-obligate parasite of cocoa. It is a windborne, leaf-penetrating, vascular pathogen, and may have evolved as an endophyte on an as yet unidentified indigenous host. The rate of disease spread on cocoa is limited because basidiocarps develop only on fresh leaf scars during wet weather, and basidiospores remain viable for a few hours on the night they are shed. Consequently, very few new infections occur beyond 80-m from diseased trees. Transmission of the disease through seed or infected cuttings has not been demonstrated. Strict quarantine measures applied to the movement of intact plants are crucial in reducing spread of the disease. Integrated management, including the planting of less susceptible genotypes, nursery construction and management, canopy pruning and shade management, provides effective control.
\end{abstract}

\section{HISTORY, DISTRIBUTION, AND IMPACT}

A devastating disease named vascular-streak dieback (VSD) was distinguished from the various dieback syndromes of cocoa induced by environmental factors and insects in Papua New Guinea (PNG) in the 1960s $(6,8)$. It caused heavy losses of mature trees and seedlings planted near older cocoa. The disease was later shown to be caused by a new genus and species of basidiomycete, Oncobasidium theobromae $(5,11)$.

VSD has since been found in most cocoa-growing areas in South and Southeast Asia and PNG, from New Britain in the east to Hainan Island, China in the north and Kerala State, India, in the west. It has been a major problem in the large commercial plantations in West Malaysia and Sabah. It is widespread in Indonesia, including in the fine flavor cocoa plantations in East and West Java, and in the large areas of newer cocoa plantings in Sulawesi. It has also been reported from southern Thailand, Burma, Vietnam, and the southern Philippines. The only known host other than cocoa is avocado, which is also an exotic plant in Southeast Asia and the Pacific (1). It is believed that the fungus evolved on an as yet unidentified indigenous host in Southeast Asia/Melanesia and transferred to introduced cocoa. Thus, VSD is another example of a new encounter disease in cocoa.

Corresponding author: D. Guest; E-mail address: d.guest@usyd.edu.au

* The $\boldsymbol{e}$-Xtra logo stands for "electronic extra" and indicates that the online version contains supplemental material not included in the print edition. Figures 1 to 5 appear in color online.

doi:10.1094/PHYTO-97-12-1654

(C) 2007 The American Phytopathological Society

\section{DISEASE SYMPTOMS AND PATHOGEN BIOLOGY}

The most characteristic initial symptom of VSD is the chlorosis of a single leaf, usually on the second or third flush behind the shoot apex, with scattered islets of green tissue 2- to $5-\mathrm{mm}$ in diameter (5) (Fig. 1A). In some areas (notably Vietnam and Sulawesi) and on some cocoa genotypes, marginal leaf necrosis appears as an atypical leaf symptom (Fig. 1B). Affected leaves are shed within a few days and symptoms progressively develop in adjacent leaves up and down the stem. Lenticels on infected trees usually become enlarged, causing roughening of the bark (Fig. 2). Three blackened vascular traces are visible when the dry surface is scraped off scars resulting from the fall of diseased leaves (Fig. 3). When stems are split, infected xylem is visible as dark streaks within the vascular tissue (Fig. 4). Eventually leaf fall occurs right to the growing tip, which then dies. Lateral buds may proliferate, but then die, causing 'broomstick' symptoms to develop (Fig. 5). The fungus may spread internally to other branches and usually kills the tree if it spreads to the trunk.

When an infected leaf falls during wet weather, hyphae may emerge from the leaf scar and develop into a basidiocarp. This structure is visible as a white, flat, velvety coating over the leaf scar and adjacent bark (Fig. 2). Basidia develop on the basidiocarp after evening rainfall. Formation and forcible discharge of basidiospores occurs mainly after midnight into the early morning. Basidiospores only remain viable for a few hours into the morning. Basidiocarps remain fertile for about a week on attached branches, but only for a day or two on cut branches. Extended periods of wetness are required for basidiocarp formation, basidiospore release, dissemination, and infection of leaves. Thus, there is a critical link between rainfall peaks and infection periods (4). 
Basidiospores germinate and hyphae penetrate unhardened leaves at branch termini by growing directly through the cuticle, above leaf veins (7). Infected leaves do not show symptoms for 3 to 5 months, by which time the pathogen has ramified through the xylem in the adjacent stem. This incubation period explains why first symptoms usually appear on the second or third flush behind the growing tip, and why peaks in the appearance of disease symptoms often occur 3 to 5 months after seasonal rainfall peaks.

\section{EPIDEMIOLOGY}

Basidiospores are dispersed by wind, although effective spore dispersal is probably limited to a few hours on the morning of their discharge when humidity is high (4). As a result, very few infections occur beyond $80 \mathrm{~m}$ from diseased cocoa. The rate of disease spread is also limited by the relatively low sporulation rate of the fungus. Basidiocarps only develop when leaf fall occurs during wet weather. Those that are formed have a short life span and only release spores at night, and then only after afternoon and evening rains. The rate of symptom development is closely correlated with rainfall incidence during a few prior months and, in PNG and West Malaysia, the disease is most common in wetter regions where annual rainfall exceeds $2,500 \mathrm{~mm}$. The disease is most damaging in seedlings less than 10 months old, which are killed by infection of the single stem. Only the most susceptible genotypes are killed by infections beginning in outer branches on older trees (4).
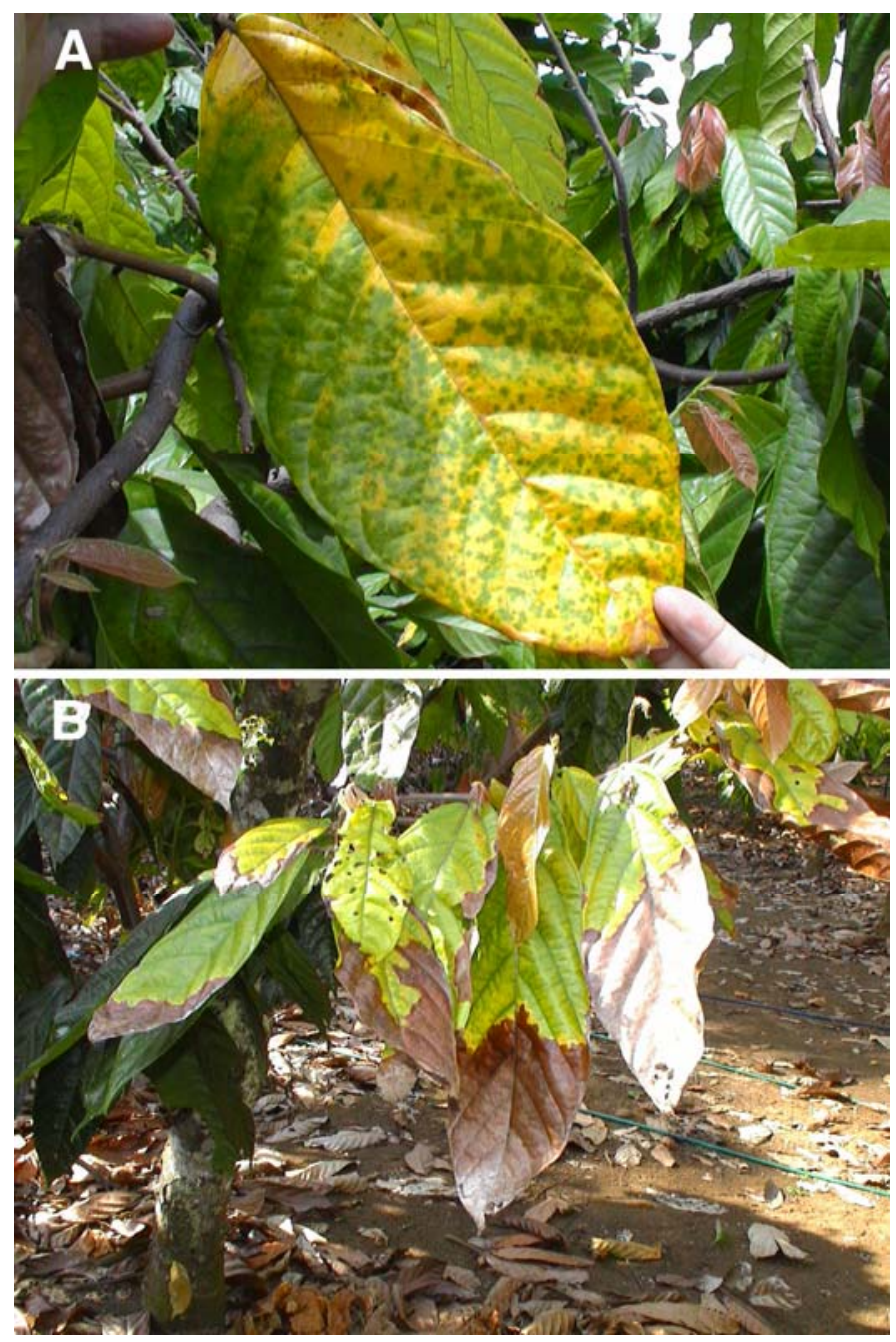

Fig. 1. Leaf symptoms of vascular-streak dieback in Vietnam. A, Typical "green island" symptom, B, atypical marginal leaf necrosis.

\section{MANAGEMENT}

Quarantine. Because natural spread of $O$. theobromae is very restricted, quarantine measures to restrict transport of the fungus by humans reduce long-distance spread of the disease. Moreover, appropriate quarantine precautions based on our current understanding of the biology and transmission of the pathogen can allow dissemination of cocoa planting material while excluding the pathogen. For example, an extremely strict quarantine procedure instituted in PNG, in the 1970s, enabled transfer of superior clones from New Britain to the disease-free islands of the North Solomons and New Ireland (9).

Extensive tests of seed collected from pods on infected branches have not been able to demonstrate transmission of the disease through seed (5). It is rare for mature pods to be formed on diseased branches, and even when they are, colonization of the placenta or embryo has not been observed. There is minimal chance of transmission through live seed, and there is no chance of transmission through fermented and dried beans. Thus, it is highly unlikely that seeds pose a threat to importing countries.

Budwood taken from infected branches does not form basidiocarps, and grafts prepared from infected budwood do not take. It is thus highly unlikely that infection will spread through grafting. Nursery seedlings can be protected by any structure that prevents foliage becoming wet for more than an hour or so, as this prevents infection and sporulation.

Disease resistance. Detailed studies of resistance are hampered by the difficulty in obtaining regular supplies of inoculum for bioassays, as the fungus cannot be maintained in culture and field-collected spores are not always available.

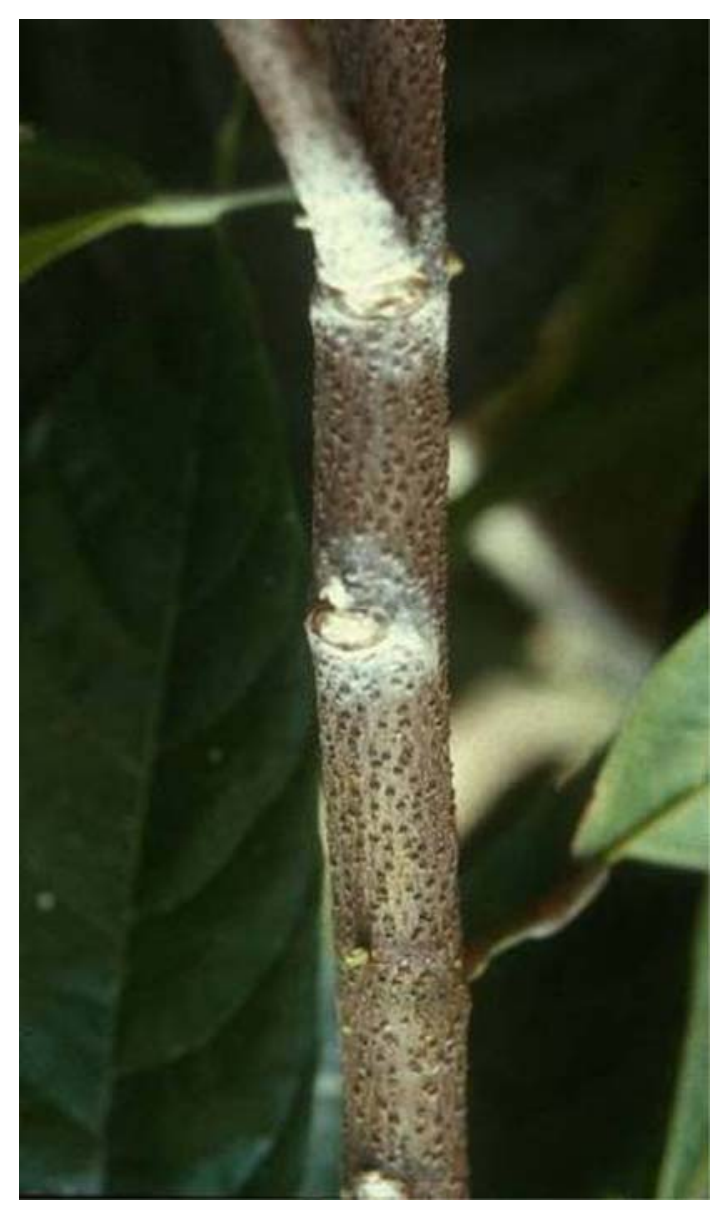

Fig. 2. Swollen lenticels and basidiocarps of Oncobasidium theobromae forming on the leaf abscission scars on a cacao branch affected by vascular-streak dieback in Sumatra, Indonesia (photo by P. D. Turner). 
The VSD epidemic in PNG during the 1960s devastated plantings established during the previous decade, killed mature trees, and a high proportion of young seedlings. The epidemic slowed by the 1970 s as the most susceptible genotypes succumbed to VSD. Selection for resistance to the disease occurred among seedlings in the heterogeneous, partly-out breeding Trinitario population of cacao in PNG (7). Also, Upper Amazon clones were

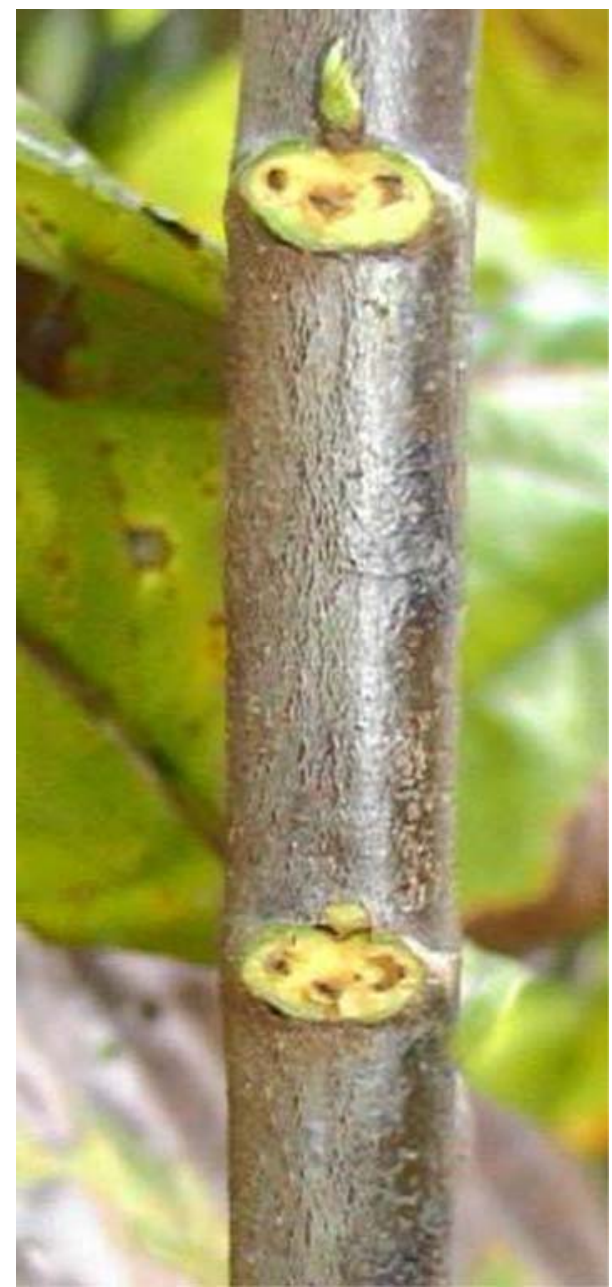

Fig. 3. Discoloration of the vascular traces on leaf abscission scars on a cacao branch affected by vascular-streak dieback.

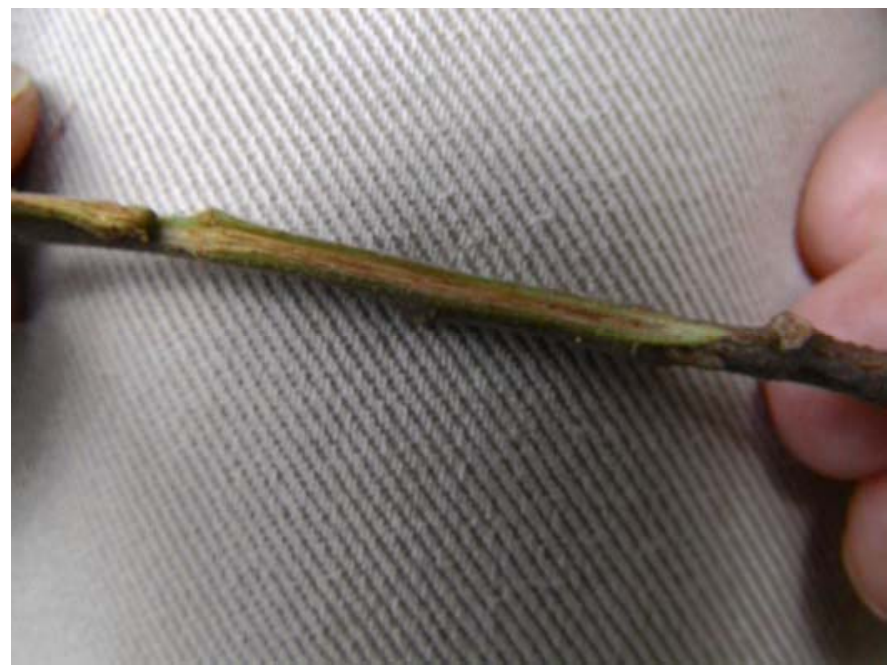

Fig. 4. Vascular streaking on a cacao branch affected by vascular-streak dieback. screened by exposure to natural epidemics in PNG and those survivors were subsequently used as male parents together with Trinitario female parents to produce hybrids having a useful degree of resistance. These are now widely grown throughout PNG.

Less susceptible genotypes still become infected, but symptoms are less severe and the pathogen grows more slowly and only rarely sporulates; invasion into larger branches and the main trunk also appears to be restricted in these genotypes. Resistance is durable and is inherited quantitatively (12). In PNG, breeding with surviving genotypes has been very successful, and has relegated VSD to minor importance in most years. Cacao in Sulawesi also appears to have a degree of resistance to VSD, probably because of natural selection operating in the genetically diverse cacao germplasm there. The disease occurs very commonly in the outer branches but rarely kills the trees.

Cultural practices. Clean nursery stock raised well away from diseased cocoa ensures that stock transplanted into the field is disease-free. Nursery stock can be further protected by growing it in a shade house or under a shelter that keeps leaves dry for all but a few hours following watering. Very high levels of infection and subsequent death have been observed in unprotected plants raised near or under older, diseased cocoa.

Pruning diseased material about $30-\mathrm{cm}$ below discolored xylem prevents further extension of infections and reduces inoculum levels by removing potential sites of sporulation. In a mature planting in Java, where highly trained teams detected and pruned out infected branches every 2 weeks for nearly 2 years, the incidence of infected trees was kept below $1 \%$. In contrast, in an unpruned planting, disease incidence increased from about 30 to $90 \%$ in a 10 -month period (10).

Shade and canopy management to increase aeration and sunlight on the foliage is of critical importance as sporulation and infection require moist conditions (2). These practices are best combined with integrated management practices designed to control Phytophthora palmivora and other pests and diseases.

Biological control. $O$. theobromae is a vascular pathogen that infects immature leaves. Potential exists to investigate the use of epiphytic microbes to reduce leaf infection, and endophytic fungi and bacteria to protect against vascular colonization. Biological control agents could also be targeted at the basidiocarp stage.

Fungicides. Systemic ergosterol biosynthesis inhibiting fungicides, including flutriafol, hexaconazole, propiconazole, tebuconazole, and triadimenol, have been successfully used to control VSD under experimental conditions. However, none of these fungicides have been shown to be commercially viable in established cocoa plantations (3; D. Guest, unpublished data).

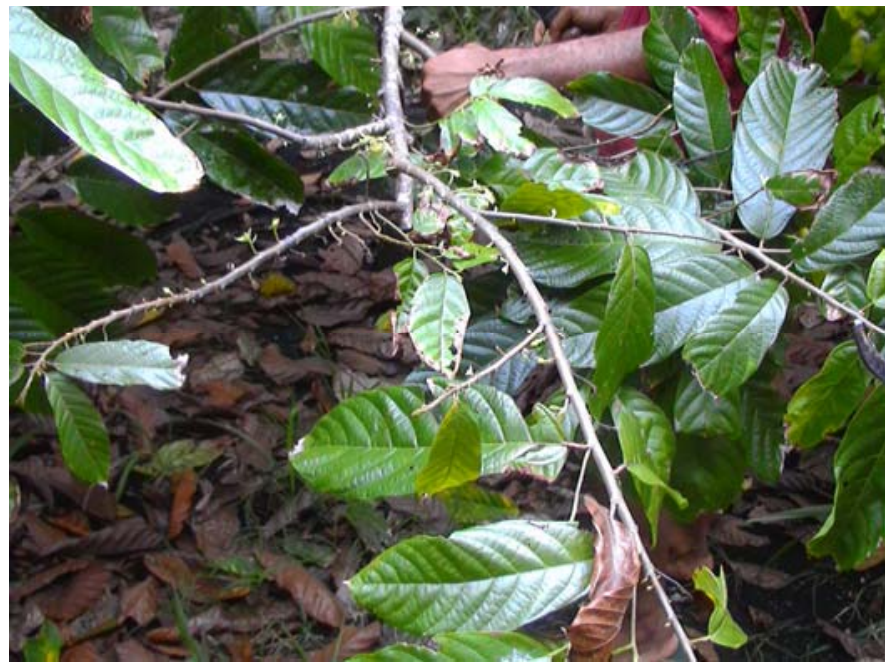

Fig. 5. "Broomsticking" caused by successive leaf drop and axillary bud development on a cacao branch affected by vascular-streak dieback. 


\section{CONCLUSION}

Integrated management, including the production of diseasefree plants in the nursery, canopy management and regular pruning of infected branches, and use of moderately resistant genotypes of cocoa provides adequate management of VSD. However, there has been an upsurge in the incidence of VSD in PNG, which has been associated with the occurrence of wetter conditions. There has also been a recent upsurge in the disease in Java, and weather conditions and poor management are probably responsible. Thus, while these upsurges do not appear to be due to a breakdown in disease resistance, this situation requires close monitoring.

In the eastern provinces of Indonesia where cocoa production has expanded rapidly, it is important that resistance to VSD be incorporated into the planting material. Following a rapid expansion of cacao planting, the disease has not been as destructive in Sulawesi as it was in PNG in the 1960s. The introduction of hybrid cacao, including Upper Amazon, Trinitario and Amelonado types, has resulted in great genetic diversity of cacao in the region; some genotypes appear to have adequate levels of resistance to the disease with potential for further selection for resistance.

\section{LITERATURE CITED}

1. Anderson, R. D. 1989. Avocado, an alternate host for Oncobasidium theobromae. Aust. Plant Pathol. 18:96-97.

2. Dennis, J. J. C., and Keane, P. J. 1992. Management strategies for control of vascular-streak dieback of cocoa. Cocoa Growers' Bull. 45:31-44.

3. Holderness, M. 1990. Control of vascular-streak dieback of cocoa with triazole fungicides and the problem of phytotoxicity. Plant Pathol. 39:286-293.

4. Keane, P. J. 1981. Epidemiology of vascular-streak dieback of cocoa. Ann. Appl. Biol. 98:227-241.

5. Keane, P. J., and Prior, C. 1991. Vascular-Streak Dieback of Cocoa. Phytopathological Papers No. 33. CAB International, Wallingford, UK.

6. Keane, P. J., Flentje, N. T., and Lamb, K. P. 1972. Investigation of vascularstreak dieback of cocoa in Papua New Guinea. Aust. J. Biol. Sci. 25:553-564.

7. Prior, C. 1979. Resistance of cocoa to vascular-streak dieback disease. Ann. Appl. Biol. 92:369-376.

8. Prior, C. 1980. Vascular-streak dieback. Cocoa Growers' Bull. 29:21-26.

9. Prior, C. 1985. Cocoa quarantine: Measures to prevent the spread of vascular-streak dieback in planting material. Plant Pathol. 34:603-608.

10. Soekirman, P., and Agus, P. 1992. Occurrence and control of VSD in Java and South-East Sulawesi. Pages 209-213 in: Cocoa Pest and Disease Management in South-East Asia and Australasia. P. J. Keane and C. A. J. Putter, eds. FAO Plant Production and Protection Paper No. 112. FAO, Rome, Italy.

11. Talbot, P. H. B., and Keane, P. J. 1971. Oncobasidium, a new genus of tulasnelloid fungi. Aust. J. Bot. 19:203-206.

12. Tan, G. Y., and Tan, W. K. 1987. Genetic variation in resistance to vascular-streak dieback in cocoa. Theor. Appl. Genet. 75:761-766. 